\title{
Prognostic value of tissue factor in patients with abdominal aortic and iliac arterial aneurysms - preliminary study
}

\author{
Jan Skóra', Tomasz Dawiskiba', Patrycja Zaleska', Jacek Kurcz², Agnieszka Mastalerz-Migas ${ }^{3}$, \\ Rajmund Adamiec ${ }^{4}$, Izabela Gosk-Bierska ${ }^{4}$
}

1Department of Vascular, General and Transplantation Surgery, Wroclaw Medical
University, Poland
'Department of General Radiology, Interventional Radiology and Neuroradiology,
Wroclaw Medical University, Poland
3Department of Family Medicine, Wroclaw Medical University, Poland
4Department of Angiology, Hypertension and Diabetology, Wroclaw Medical University,
Poland

Submitted: 12 June 2013

Accepted: 5 August 2013

Arch Med Sci 2013; 9, 6: 1071-1077

DOI: 10.5114/aoms.2013.39795

Copyright @ 2013 Termedia \& Banach

\begin{abstract}
Introduction: The decision on the time and choice of strategy of treatment of abdominal aortic aneurysm must be especially carefully balanced. The aim of the study was to evaluate the tissue factor (TF) plasma level as a potential factor useful in anticipation of abdominal aortic aneurysm and/or iliac arterial aneurysm via comparison of plasma TF level in patients with ruptured and non-ruptured aneurysms. Material and methods: The study included 33 patients with aneurysm (17 operated on electively because of non-ruptured aneurysm and 16 operated on emergently due to ruptured aneurysm), 33 claudicant patients with atherosclerosis of the abdominal aorta and iliac arteries with normal diameter of arteries, and 30 healthy controls. Plasma TF level was assessed by ELISA method using the IMUBIND Tissue Factor ELISA Kit (American Diagnostica Inc.).

Results: The study showed an increased TF level in patients with aneurysm (134 $\pm 54 \mathrm{pg} / \mathrm{ml}$ ) and in patients with atherosclerosis without concomitant aneurysm $(91 \pm 30 \mathrm{pg} / \mathrm{ml})$ in comparison with the control group $(62 \pm 20 \mathrm{pg} / \mathrm{ml})$, respectively $p<0.001$ and $p=0.008$. A significantly higher TF plasma level was observed in patients with ruptured abdominal aortic aneurysms $(160 \pm 57 \mathrm{pg} / \mathrm{ml})$ as compared to patients with non-ruptured aortic aneurysms $(109 \pm 39 \mathrm{pg} / \mathrm{ml})$ or peripheral arterial occlusive disease $(91 \pm 30 \mathrm{pg} / \mathrm{ml})$, respectively $p<0.001$ and $p<0.001$. The difference in TF level between the group with non-ruptured aortic aneurysms $(109 \pm 39 \mathrm{pg} / \mathrm{ml})$ and the patients with atherosclerosis without aneurysm ( $91 \pm 30 \mathrm{pg} / \mathrm{ml}$ ) was not statistically significant.

Conclusions: No difference in TF level between patients with non-ruptured AAA/IAA and patients with aortic and iliac atherosclerosis without aneurysm indicates that an increased TF plasma level is not specific for any of the abovementioned vascular pathologies.
\end{abstract}

Key words: tissue factor, ruptured, non-ruptured aneurysm, abdominal aorta, iliac artery.

\section{Introduction}

Abdominal aortic aneurysm (AAA) and iliac arterial aneurysms (IAA) are among the main reasons for vascular surgical procedures/interventions of

\author{
Corresponding author: \\ Agnieszka Mastalerz-Migas \\ $\mathrm{MD}, \mathrm{PhD}$ \\ Department \\ of Family Medicine \\ Wroclaw Medical University \\ 1 Syrokomli St \\ 51-141 Wroclaw, Poland \\ Phone: +48 713255126 \\ E-mail: \\ agnieszka.migas@gmail.com
}


aorta and iliac arteries. On the basis of previous epidemiological studies AAA/IAA was demonstrated in $4-10 \%$ of people over $75[1,2]$. The result of treatment of AAA and/or IAA depends on many factors, e.g. sex, age, smoking, comorbidities, methods of treatment, experience of the vascular surgery center, as well as activity of the coagulation-fibrinolysis system [3]. Detailed analysis of such factors significantly facilitates prognosis of the postoperative clinical course including risk of death [1-3]. Determinant prognostic factors are aneurysmal size and localization. Elective surgical or endovascular treatment is indicated in the case of AAA of diameter more than $5.5 \mathrm{~cm}$ and in the case of rapidly growing (more than $0.6 / 7 \mathrm{~cm}$ per 6 months or more than $1 \mathrm{~cm}$ per year) so-called small aneurysms (size $<5 \mathrm{~cm})[1,2]$. Surgical treatment of IAA is indicated in the case of aneurysm diameter exceeding $3.5 \mathrm{~cm}$ and/or rapid growth (more than $3 \mathrm{~mm}$ per year) [4]. Due to anatomical location the detection rate of IAA by means of abdominal ultrasound is much lower when compared with AAA.

The etiology of aneurysms is not well known. Histologically the wall of AAA is characterized by chronic inflammation, reduction in the number of smooth muscle cells, destruction of elastin fibers and remodeling of extracellular matrix, activation of platelets and plasmatic coagulation [5-8]. An atherosclerotic component is present in most cases of AAA. An association between atherosclerosis of both coronary arteries and peripheral arterial vessels and the presence of AAA was demonstrated; nevertheless the mechanism of the phenomenon is not well researched $[9,10]$. Some studies indicate the occurrence of AAA in response to the atherosclerotic process and vascular wall remodeling [11, 12]. Rupture of AAA is equivalent to extensive vascular wall damage and release of a large amount of tissue factor. Tissue factor (TF, thromboplastin) is crucial in induction and enhancement of the coagulation process. It also plays a role as a cofactor essential to activate factor VII. Complex TF/VIla activates factors IX and X, thus initiating the cascade of proteolytic reactions that lead to generation of thrombin and clot formation [13]. Apart from a key role in the coagulation process, TF participates inter alia in inflammatory reactions and angiogenesis [14, 15]. The physiologically low level of TF is increased in clinical conditions associated with damage of vessel endothelium and other tissues depending on the degree of vascular wall damage [16]. The utilization of TF level as a potential marker for progression and/or risk of rupture of AAA could be of significance in early diagnosis of AAA rupture. Previous studies have demonstrated the increase of TF in the course of traumas, infections, cardiovascular disorders, diabetes, venous thrombosis and neoplasms [17-21]. The atherosclerotic process involving the aorta and peripheral arteries resulting in chronic lower limb ischemia seems to be a factor independent from diabetes, arterial hypertension, obesity, lipid disorders, age and sex that contributes to an increase in plasma TF level [18]. A few reports on TF and its physiological inhibitor tissue factor pathway inhibitor (TFPI) in patients with AAA point to their participation in formation of perimural thrombus in the aneurysmal lumen [22-24].

The purpose of the study was to evaluate TF level as a potential factor useful in anticipation of AAA and/or IAA rupture via comparison with plasma TF concentration in patients with ruptured and nonruptured aneurysms. Considering that most of the patients with AAA demonstrate atherosclerotic lesions in both the abdominal aorta and iliac arteries and the atherosclerotic process is a factor causing increase in TF concentration, it was planned to compare the parameter (TF level) between patients with AAA and/or IAA and patients with atherosclerosis of the abdominal aorta and iliac arteries presenting symptoms of chronic lower limb ischemia (stage 2 according to Fontaine classification) and with normal vessel diameter.

\section{Material and methods}

The analyzed group included: 33 patients with AAA and/or IAA treated operatively between 20092012 in the Department of Vascular, General and Transplantation Surgery of Wroclaw Medical University; 33 patients with atherosclerosis of the abdominal aorta and iliac arteries with normal diameter of arteries, presenting symptoms of peripheral arterial occlusive disease (PAOD) grade II according to Fontaine and treated medically in the Department of Angiology, Hypertension and Diabetology of Wroclaw Medical University; and the control group of 30 healthy volunteers, selected from among employees of Wroclaw Medical University. Among 33 patients with AAA and/or IAA treated surgically, 17 patients were operated on electively because of non-ruptured aneurysm and 16 patients were operated on emergently due to ruptured aneurysm. The protocol of this study was approved by the Commission of Bioethics at the Wrocław Medical University.

The diagnosis of AAA and/or IAA was established based on CT-angiography. The diagnosis of chronic lower limb ischemia in the course of atherosclerosis of the aorta and iliac arteries was made on the basis of history of claudication, physical examination, segmental blood pressure measurements (ankle-brachial index < 0.8) and duplex-Doppler examination.

In all the patients plasma TF level was measured. In healthy controls and in subgroups treated electively, blood samples were taken after overnight fasting. In patients with ruptured AAA blood samples were taken at admission to hospital. 
The $5 \mathrm{ml}$ blood samples were drawn from an antecubital vein and transferred to a test tube with $3.2 \%$ sodium citrate in the proportion $9: 1$, and centrifuged at 3,000 $\times \mathrm{g}$ for $15 \mathrm{~min}$. Plasma was stored at $80^{\circ} \mathrm{C}$. Plasma TF level was assessed by enzymelinked immunosorbent assay (ELISA) using a commercially available kit: IMUBIND Tissue Factor ELISA Kit no. 845 (American Diagnostica Inc., Stamford).

\section{Statistical analysis}

The collected data were analyzed statistically using the Statistica 10 software package (StatSoft, Poland). Statistical significance of between-group differences was assessed using Student's t-test (quantitative variables) and $\chi^{2}$ analysis (qualitative variables).

The Mann-Whitney $U$-test was used to compare subgroups of patients. As the data were not normally distributed, Spearman's rank test was used to correlate TF concentration and clinicopathological variables. Value of $p<0.05$ was considered statistically significant. All the data are presented in the tables as mean values \pm standard deviation.

\section{Results}

Demographic and clinical data of analyzed groups are compared in Table I. Mean age in the subgroup with aortic and iliac arterial atherosclerosis was comparable with the control subgroup. However, mean age in patients with AAA and/or IAA was significantly higher in comparison with both the aortic/iliac atherosclerosis subgroup and healthy control. Males comprised at least $80 \%$ of patients in all the subgroups. Arterial hypertension was shown to be more frequent in the AAA/IAA subgroup than in the subgroup with aortic/iliac arterial atherosclerosis and normal arterial diameter. On the other hand, patients with aortic/iliac arterial atherosclerosis were often heavy smokers. No significant differences in incidence of diabetes and lipid disorders were demonstrated in analyzed groups.

The comparison of risk factors and clinical course between patients with ruptured and non-ruptured AAA and/or IAA is shown in Table II. No significant differences referring to the size of ruptured and non-ruptured aneurysms were demonstrated. Perimural thrombi were detected in the lumen of all the aneurysms.

Seventy-five percent of patients with ruptured AAA and/or IAA presented symptoms of circulatory insufficiency and/or hypovolemic shock. Myocardial infarction was the most common complication in the early postoperative period $-18.8 \%$.

Table I. Demographic data and risk factors of atherosclerosis in analyzed groups

\begin{tabular}{|c|c|c|c|c|c|}
\hline Parameter & $\begin{array}{c}\text { AAA/IAA } \\
\text { Total }(n=33)\end{array}$ & $\begin{array}{c}\text { AAA/IAA } \\
\text { Non-ruptured }(n=17)\end{array}$ & $\begin{array}{l}\text { AAA/IAA Ruptured } \\
\quad(n=16)\end{array}$ & $\begin{array}{l}\text { PAOD } \\
(n=33)\end{array}$ & $\begin{array}{l}\text { Control } \\
(n=30)\end{array}$ \\
\hline \multicolumn{6}{|l|}{ Age, $n(\%)$ [years] } \\
\hline Median & $69.6 \pm 9.3$ & $67.8 \pm 9.6$ & $71.7 \pm 8.9$ & $58.9 \pm 6.9$ & $54.4 \pm 7.6$ \\
\hline Range & $52-89$ & $52-84$ & $57-89$ & $48-78$ & $46-71$ \\
\hline \multicolumn{6}{|l|}{ Sex, $n(\%)$} \\
\hline Men & $28(84.8)$ & $14(82.4)$ & $14(87.5)$ & $27(81.8)$ & $24(80)$ \\
\hline Women & $5(15.2)$ & $3(17.6)$ & $2(12.5)$ & $6(18.2)$ & $6(20)$ \\
\hline Smoking, $n(\%)$ & $19(57.6)$ & $12(70.6)$ & $7(43.7)$ & $32(93.9)$ & 0 \\
\hline Currently & $7(21.2)$ & $5(29.4)$ & $2(12.5)$ & $18(54.5)$ & \\
\hline In the past & $12(36.4)$ & $7(41.2)$ & $5(31.2)$ & $13(39.4)$ & \\
\hline Arterial hypertension, $n(\%)$ & $29(87.8)$ & $17(100)$ & $12(75)$ & $20(60.6)$ & 0 \\
\hline Diabetes, $n(\%)$ & $11(33.3)$ & $6(35.3)$ & $5(31.2)$ & $11(33.3)$ & 0 \\
\hline Lipid disorders, $n$ (\%) & $20(60.6)$ & $11(64.7)$ & $9(56.2)$ & $19(57.6)$ & 0 \\
\hline Coronary disease, $n(\%)$ & $9(27.3)$ & $5(29.4)$ & $4(25.0)$ & $12(36.4)$ & 0 \\
\hline Carotid artery disease, $n(\%)$ & $7(21.2)$ & $5(29.4)$ & $2(12.5)$ & $19(57.6)$ & 0 \\
\hline Renal failure, $n$ (\%) & $3(9.1)$ & $1(5.9)$ & $2(12.5)$ & $0(0)$ & 0 \\
\hline \multicolumn{6}{|l|}{ Pharmacotherapy, $n$ (\%) } \\
\hline Calcium channel blockers & $11(33.3)$ & $6(35.3)$ & $5(31)$ & $12(36.4)$ & 0 \\
\hline$\beta$-blockers & $17(51.5)$ & $9(53)$ & $8(50)$ & $11(33.3)$ & 0 \\
\hline Statins & $22(66.7)$ & $10(58.8)$ & $12(75)$ & $30(90.9)$ & 0 \\
\hline Antiplatelet drugs & $20(60.6)$ & $11(64.7)$ & $6(56.2)$ & $29(87.9)$ & 0 \\
\hline Anticoagulants (warfarin) & $4(12.1)$ & $3(17.6)$ & $1(6.2)$ & $3(9.1)$ & 0 \\
\hline
\end{tabular}


Table II. Clinical profile of patients treated surgically due to non-ruptured and ruptured abdominal aortic and/or iliac arterial aneurysms

\begin{tabular}{|c|c|c|}
\hline Variable & $\begin{array}{l}\text { Non-ruptured AAA/IAA } \\
\qquad(n=17)\end{array}$ & $\begin{array}{l}\text { Ruptured AAA/IAA } \\
\qquad(n=16)\end{array}$ \\
\hline \multicolumn{3}{|l|}{ Localization of aneurysms, $n$ (\%) } \\
\hline Only abdominal aorta & $12(70.6)$ & $8(50)$ \\
\hline Only iliac arteries & $2(11.8)$ & $2(12.5)$ \\
\hline Both abdominal aorta and iliac arteries & $3(17.6)$ & $6(37.5)$ \\
\hline \multicolumn{3}{|c|}{ Morphologic evaluation of an aneurysm, mean (range) $[\mathrm{cm}]$} \\
\hline Diameter of abdominal aortic aneurysm & $6.26(3.6-7.8)$ & $6.77(5-12.1)$ \\
\hline Diameter of iliac arterial aneurysm & $3.2(2-62)$ & $5.35(1.8-13)$ \\
\hline Thrombus in aneurysmal lumen, $n$ (\%) & $17(100)$ & $100(16)$ \\
\hline Thickness of thrombus, mean (range) $[\mathrm{cm}]$ & $2.94(1.8-5)$ & $2.59(1-7)$ \\
\hline \multicolumn{3}{|l|}{ Type of surgery, $n(\%)$} \\
\hline Straight prosthesis & $10(58.8)$ & $7(43.8)$ \\
\hline Bifurcated prosthesis & $4(23.5)$ & $6(37.5)$ \\
\hline Stent graft & $2(11.8)$ & $2(12.5)$ \\
\hline Iliac-femoral bypass & $1(5.9)$ & $1(6.30)$ \\
\hline Time of surgery, mean (range) [h] & $2.35(1.4-3.2)$ & $2.28(1.2-3.25)$ \\
\hline \multicolumn{3}{|l|}{ Preoperative clinical condition, $n(\%)$} \\
\hline Hypovolemic shock & 0 & $11(68.8)$ \\
\hline Circulation failure & 0 & $12(75)$ \\
\hline \multicolumn{3}{|l|}{ Early postoperative complications, $n(\%)$} \\
\hline Death & 0 & $7(43.8)$ \\
\hline Myocardial infarction & 0 & $3(18.8)$ \\
\hline Renal failure & 0 & $1(6.3)$ \\
\hline Acute lower limb ischemia & 0 & $2(12.5)$ \\
\hline Intestinal necrosis & 0 & $2(12.5)$ \\
\hline Paraplegia due to spinal cord ischemia & 0 & $1(6.3)$ \\
\hline
\end{tabular}

The other early postoperative complications in patients with ruptured AAA/IAA included acute kidney failure, acute lower limb ischemia, intestinal necrosis and paraplegia due to spinal cord ischemia $-6.3 \%, 12.5 \%, 12.5 \%$ and $6.3 \%$ respectively. Such complications were the causes of death in over $40 \%$ of the subgroup. None of the above-mentioned complications occurred in patients with non-ruptured aneurysms treated electively. The most common localization of aneurysms was the abdominal aorta; therefore implantation of a straight vascular prosthesis was the prevailing surgical management.

No correlation between smoking and risk of aneurysm rupture was demonstrated. No correlation between TF level and either aneurysmal size or perimural thrombus size was found.

The TF level was significantly higher both in patients with AAA/IAA $(134 \pm 54 \mathrm{pg} / \mathrm{ml})$ and in patients with atherosclerotic lesions $(91 \pm 30 \mathrm{pg} / \mathrm{ml})$ when compared with the control group $(62 \pm 20 \mathrm{pg} / \mathrm{ml})$, respectively $p=0.000107$ and $p=0.008428$. Furthermore, the TF level was significantly higher in patients with AAA/IAA when compared with the subgroup with atherosclerotic aortic changes $(p<0.001)$. Further analysis demonstrated that the difference resulted from the very high TF level observed in patients with ruptured aneurysms. The level of TF in patients with ruptured aneurysms $(160 \pm 57 \mathrm{pg} / \mathrm{ml})$ was significantly higher in comparison with both the non-ruptured aneurysm subgroup (109 $\pm 39 \mathrm{pg} / \mathrm{ml}$ ) and the aortic atherosclerotic lesions subgroup (91 $\pm 30 \mathrm{pg} / \mathrm{ml})$, respectively $p<0.001$ and $p<0.001$. No significant difference in TF level was observed between patients with non-ruptured aneurysms $(109 \pm 39 \mathrm{pg} / \mathrm{ml})$ and patients with aortic/iliac arterial atherosclerotic lesions and normal arterial diameter $(91 \pm 30 \mathrm{pg} / \mathrm{ml})$.

The analysis of TF level in particular groups is shown in Tables III and IV. 


\section{Discussion}

The decision on the time and choice of strategy of treatment of AAA and/or IAA must be especially carefully balanced, because both medical management burdened with the risk of aneurysm rupture, and invasive treatment associated with increased risk of myocardial infarction, cerebral stroke, renal failure, and perioperative bleeding, are related to a high risk of death. To improve the clinical course and to follow up the results of AAA surgery, studies are conducted in search of biological markers that evaluate the risk of postoperative complications $[25,26]$ and predict the aneurysmal size progression [27].

On the basis of a meta-analysis of previous studies, Golledge et al. mentioned biomarkers associated with progression of AAA size (elastin peptides, $\mathrm{N}$-terminal propeptide of type III procollagen (PIIINP), elastase, $\alpha 1$ antitrypsin, plasmin-antiplasmin complex, tissue plasminogen activator, homocysteine, urokinase plasminogen activator, osteopontin, interferon $\gamma$, macrophage migratory inhibitor factor, transforming growth factor, C-reactive protein) and biomarkers related to the clinical course, including aortic rupture (fibrinogen, $\alpha 1$-antitrypsin) [28]. There are no studies on evaluation of TF as a prognostic factor in relation to progression of size and risk of rupture of AAA. Previous studies devoted to the process of coagulation and fibrinolysis in patients with AAA related first of all to the pathomechanism of formation of lesions in the aneurysmal vascular wall, taking the susceptibility of formation of perimural thrombus into special consideration [9, 22, 23]. The conclusions concerning the correlation between activation of the coagulation/fibrinolysis process and the occurrence of AAA are based primarily on the preoperative assessment of non-ruptured aneurysms. Yamazumi et al. stated that TF level in 36 patients undergoing elective surgery due to non-ruptured aneurysms does not depend on the aneurysm size and does not correlate with other exponents of the hemostasis process. However, the researchers observed a correlation between TFPI level and aneurysmal size and levels of TAT, D-dimers, fibrinogen degradation products, and $\alpha 2$ plasminogen inhibitor [29]. Nomura et al. demonstrated significantly higher levels of TFPI, TAT, D-dimers, and sICAM-1 in 17 patients with non-ruptured AAA in comparison with a control group of healthy volunteers of comparable age. According to the authors, a positive correlation between AAA size and levels of TAT, D-dimers, and sICAM-1 indicates the possibility to make use of such parameters in diagnosis and follow-up of AAA progression [24]. According to Kotschy et al., increase of TF and TAT levels without simultaneous increase of the TFPI level in patients with non-ruptured aneurysms may induce a proteolytic process
Table III. TF level in particular groups

\begin{tabular}{|lc|}
\hline Group & TF level $[\mathrm{pg} / \mathrm{ml}]$ \\
\hline AAA/IAA $(n=33)$ & $134.6 \pm 54.7$ \\
\hline Ruptured AAA/IAA $(n=16)$ & $160.7 \pm 57.7$ \\
\hline Non ruptured AAA/IAA $(n=17)$ & $109.9 \pm 39.3$ \\
\hline PAOD $(n=33)$ & $91.8 \pm 30.4$ \\
\hline Control $(n=30)$ & $62.2 \pm 20.9$ \\
\hline
\end{tabular}

Table IV. Statistical analysis of TF level in particular groups

\begin{tabular}{|lc|}
\hline Compared groups & Value of $p$ \\
\hline Control vs. AAA/IAA & $<0.001$ \\
\hline Control vs. ruptured AAA/IAA & $<0.001$ \\
\hline Control vs. non-ruptured AAA/IAA & 0.001 \\
\hline Control vs. PAOD & 0.008 \\
\hline Ruptured AAA/IAA vs. non-ruptured AAA/IAA & $<0.001$ \\
\hline PAOD vs. AAA/IAA & $<0.001$ \\
\hline PAOD vs. ruptured AAA/IAA & $<0.001$ \\
\hline PAOD vs. non-ruptured AAA/IAA & Non-significant \\
\hline
\end{tabular}

in the vessel wall through an increase in thrombotic susceptibility and clot formation in the aneurysmal lumen [23].

There are only a few studies, additionally performed in small groups of patients, comparing the condition of the coagulation-fibrinolysis system between ruptured and non-ruptured aneurysms. Adam et al. stated that the occurrence of AAA is accompanied by an increase in thrombotic susceptibility expressed by activation of the coagulation process and inhibition of fibrinolysis, enhanced in the case of aneurysmal rupture. Significantly higher levels of thrombin-antithrombin complex (TAT), prothrombin fragment $1+2$ (PF 1+2) and plasminogen activator inhibitor (PAI) and lower levels of tissue plasminogen activator (t-PA) were demonstrated in 10 patients with ruptured AAA in comparison with 9 patients with non-ruptured AAA [30]. This phenomenon was considered as a natural response to the condition of direct risk of severe bleeding. On the other hand, the authors concluded that the condition of hypercoagulation concurrent with aneurysm rupture is conducive to clot formation in micro- and macrocirculation and to thromboembolic complications and thus is a risk factor for infarction, stroke, multiple organ dysfunction syndrome (MODS) [30]. Only one study evaluating the influence of AAA rupture on TF level has been reported so far. Hobbs et al. performed a prospective study comparing 17 patients undergoing elective surgery due to AAA with 10 patients undergoing emergent surgery due to AAA rupture. The researchers observed a significantly higher preoperative TF level in the subgroup with ruptured AAA compared with 
patients with non-ruptured AAA. However, no difference in TFPI level was demonstrated between the preoperative period and soon after the surgery. The TFPI level in patients with ruptured AAA was observed just after $24 \mathrm{~h}$ and $48 \mathrm{~h}$ following surgery. The authors consider that preoperative lack of increase in TFPI level simultaneous with increase in TF level in patients with ruptured AAA means an increase in thrombotic susceptibility and constitutes a mechanism that reduces the risk of severe bleeding complications [31].

We found in our study a significant increase in plasma TF level both in patients with AAA and in patients with aortic and peripheral arterial atherosclerosis compared to the control group. The results are similar to those obtained in previous studies [18, 23]. The further analysis demonstrated that increase in TF level in the AAA subgroup in comparison with aortic atherosclerotic patients without AAA is associated with aneurysm rupture. In the subgroup with ruptured AAA, the tissue factor level was significantly higher than in other subgroups, including patients with non-ruptured aneurysms undergoing elective surgery. The rupture of the aneurysmal wall and enhanced release of TF into the bloodstream might be one of the reasons for this phenomenon. An additional factor that might stimulate increase in TF level might be the damage of tissues located in the retroperitoneal space and the root of the mesentery due to blood extravasated from the ruptured aneurysm [31, 32]. No difference in TF level between the subgroup with non-ruptured aneurysm and patients with atherosclerotic lesions without pathological dilatation of both aorta/iliac arteries suggests a similar degree of damage of the vascular wall that is responsible for release of thromboplastin both in the course of occlusive atherosclerosis and in the course of aortic aneurysm formation. The fact indicates that the increase in TF level in relation to the control group is not specific for either aortic atherosclerosis or aortic aneurysms and does not differentiate between these pathologies. The results of performed studies unambiguously demonstrate that high serum TF level accompanies ruptured AAAs. Due to this fact, the assessment of TF level can be used as an additional parameter to confirm the rupture of AAA. However, on the basis of our results it is difficult to evaluate unambiguously whether the observed significantly higher TF level in patients with ruptured $A A A / I A A$ as compared with the non-ruptured AAA/IAA subgroup can be adopted as a useful predictor of aortic aneurysm rupture or as a proof of occurrence of rupture of the vascular wall.

Hence further prospective randomized studies in larger groups of patients with AAA are needed.

The limited number of patients with ruptured AAA/IAA does not allow us to answer the question whether TF level correlates with complications in the form of bleeding, circulatory failure, myocardial infarction, renal failure, lower limb and visceral ischemia, and finally death. In the discussed reports TF level was evaluated at the moment of admission to hospital.

In conclusion, increased TF levels are observed both in patients with abdominal aortic aneurysm and/or iliac arterial aneurysm and in patients with abdominal aortic and iliac arterial atherosclerosis without concomitant aneurysm in comparison with the control group. At the same time, the lack of difference in TF level between patients with non-ruptured aortic and/or iliac arterial aneurysm and patients with aortic and iliac atherosclerosis without aneurysm indicates that increase of TF level is not specific for any of the above-mentioned vascular pathologies. A significantly higher TF level was observed in patients with ruptured abdominal aortic aneurysms as compared to patients with nonruptured aortic aneurysms or peripheral arterial occlusive disease. Further studies are needed to evaluate whether an increase in TF level in patients with abdominal aortic and/or iliac arterial aneurysm can be considered as a predictor of aneurysmal rupture or rather as a result of vascular wall rupture.

\section{References}

1. Blanchard JF. Epidemiology of abdominal aortic aneurysms. Epidemiol Rev 1999; 21: 207-21.

2. Wasseff M, Baxter BT, Chisholm RL, Dalman RL, Fillinger MF, Heinecke J. Pathogenesis of abdominal aortic aneurysm: a multidisciplinary research program supported by the National Heart, Lung and Blood Institute. J Vasc Surg 2001; 34: 730-8.

3. Wahlberg E, Olofsson P, Goldstone J. Emergency vascular surgery: a practical guide. Springer-Verlag, Berlin 2007; 75-91.

4. Huang Y, Gloviczki P, Duncan AA, et al. Common iliac artery aneurysm: expansion rate and results of open surgical and endovascular repair. J Vasc Surg 2008; 47: 1203-10.

5. Ailawadi G, Eliason JL, Upchurch GR Jr, Arbor A. Current concepts in the pathogenesis of abdominal aortic aneurysm. J Vasc Surg 2003; 38: 584-8.

6. Thompson RW, Geraghty PJ, Lee JK. Abdominal aortic aneurysms: basic mechanisms and clinical implications. Curr Probl Surg 2002; 39: 110-230.

7. Kadaoglou NP, Lapis CD. Matrix metalloproteinases: contribution to pathogenesis, diagnosis, survelliance and treatment of abdominal aortic aneurysms. Curr Med Res Opin 2004; 20: 419-32.

8. Knox JB, Sukhova GK, Whittemore AD, Libby P. Evidence for altered balance between matrix metalloproteinases and their inhibitors in human aortic diseases. Circulation 1997; 95: 205-12.

9. Golledge J, Norman PE. Atherosclerosis and abdominal aortic aneurysm: cause, response, or common risk factors? Arterioscler Thromb Vasc Biol 2010; 30: 1075-7.

10. Cornuz J, Sidoti Pinto C, Tevaearai H, Egger M. Risk factors for asymptomatic abdominal aortic aneurysm: systematic review and meta-analysis of population-based screening studies. Eur J Public Health 2004; 14: 343-9. 
11. Kaschina E, Scholz H, Steckelings UM, et al. Transition from atherosclerosis to aortic aneurysm in humans coincides with an increased expression of RAS components. Atherosclerosis 2009; 205: 396-403.

12. Ward MR, Pasterkamp G, Yeung AC, Borst C. Arterial remodeling. Mechanisms and clinical implications. Circulation 2000; 102: 1186-91.

13. Nemerson Y. Tissue factor and hemostasis. Blood 1988; 71: 1-8.

14. Camerer E, Huang W, Couqhlin SR. Tissue factor - and factor X-dependent activation of protease - activated receptor 2 by factor VIla. Proc Natl Acad Sci USA 2000; 97: 5255-60.

15. Mackman N. Role of tissue factor in hemostasis, thrombosis, and vascular development. Arterioscler Thromb Vasc Biol 2004; 24: 1015-22.

16. Giensen PL, Rauch U, Borhmann B, et al. Blood - borne tissue factor: another view of thrombosis. Proc Natl Acad Sci USA 1999; 96: 2311-5.

17. Gando S, Nanzaki S, Sasaki S, Kemmotsu O. Significant correlation between tissue factor and thrombin markers in trauma and septic patients with disseminated intravascular coagulation. Thromb Haemost 1998; 79: 1111-8.

18. Gosk-Bierska I, Wysokiński W, Karnicki K, Adamiec R. Tissue factor, tissue pathway factor inhibitor and risk factors of atherosclerosis In patients with chronic limbs ischemia: preliminary study. Int Angiol 2008; 27: 296-301.

19. Manly D, Boles J, Mackman N. Role of tissue factor in venous thrombosis. Annu Rev Physiol 2011; 73: 515-25.

20. Rickles FR, Patierno S, Fernandes PM. Tissue factor, thrombin, and cancer. Chest 2003; 124: 58-68S.

21. Suefuji H, Ogawa $H$, Yasue H, el al. Increased plasma tissue factor levels in acute myocardial interaction. Am Heart J 1997; 134: 253-9.

22. Cnotliwy M, Jastrzebska M, Wiernicki I, Chełstowski K. Coagulation anffibrynolysis in the abluminal layer of the thrombus within abdominal aortic aneurysym. Acta Angiol 2007; 13: 114-21.

23. Kotschy M, Witkiewicz W, Grendziak R, Dubis J, Zapotoczny N, Kotschy D. Selected clotting factors In blond of patients with abdominal aortic aneurysms. Kardiol Pol 2012; 70: 574-9.

24. Nomura F, Ihara A, Yoshitatsu M, Tamura K, Katayama A, Ihara K. Relationship between coagulation cascade, cytokine, adhesion molecule and aortic aneurysm. Eur J Cardiothorac Surg 2003; 23: 1034-9.

25. Waliszek M, Waliszek-Iwanicka A, Grycewicz T, et al. Prognostic value of plasma $\mathrm{N}$-terminal pro-B-type natriuretic peptide concentration in patients with normal and impaired left ventricular systolic function undergoing surgery for abdominal aortic aneurysm. Arch Med Sci 2011; 7: 642-7.

26. Kokot M, Biolik G, Ziaja D, et al. Endothelium injury and inflammatory state during abdominal aortic aneurysm surgery: scrutinizing the very early and minute injurious effects using endothelial markers - a pilot study. Arch Med Sci 2013; 9: 479-86.

27. Wallinder J, Bergqvist D, Henriksson A. Haemostatic markers in patients with abdominal aortic aneurysm and the impact of aneurysm size. Thromb Res 2009; 124: 423-6.

28. Golledge J, Tsao PS, Dalman RL, Norman PE. Circulating markers of abdominal aortic aneurysm presence and progression. Circulation 2008; 118: 2382-92.

29. Yamazumi K, Ojiro M, Okumura H, Aikou T. An activated state of blood coagulation and fibrinolysis in patients with abdominal aortic aneurysm. Am J Surg 1998; 175: 297-301.
30. Adam DJ, Ludlam CA, Ruckley CV, Bradbury AW. Coagulation and fibrinolysis in patients undergoing operation for ruptured and nonruptured infrarenal abdominal aortic aneurysms. J Vasc Surg 1999; 30: 641-50.

31. Hobbs SD, Haggart P, Fegan C, Bradbury AW, Adam DJ. The role of tissue factor in patients undergoing open repair of ruptured and nonruptured abdominal aortic aneurysms. J Vasc Surg 2007; 46: 682-6.

32. Adam DJ, Haggart PC, Ludlam CA, Bradbury AW. Von Willebrand factor and platelet count in ruptured abdominal aortic aneurysm repair. Eur J Vasc Endovasc Surg 2003; 26: 412-7. 